vessels were proposed.

1. It is advantage to use a "cylindrical bottle with a glass-stopper and a flat bottom as the estimating vessel in order to keep a constant contact surface during the reaction of the enzyme.

2. A vessel with a definite diameter should always be used for the comparision of the enzyme-action.

3. It is desirable to record the diameter of the estimating bottle in each experiment.

\title{
On the Nutritive Value of Synthetical Fats Containing Fatty Acids of Uneven Number of Carbon Atoms.
}

\author{
By Jun-ichi Ozakr.
}

(From the Biochemical Laboratory, College of Agriculture, Imperial University of Tokyo).

(Received Nov. 20th. 1925.)

It is a well known fact that fatty acids occurring in natural fats generally contain even-number carbon atoms. K. Takahashi (J. Chem. Soc. Japan. Vol. 43 No. 3 ) in our laboratory has recently prepared a series of triglycerides from chemically pure fatty acids, and found that, by the feeding experiments of albino rats with them, those fats consisting of lower fatty acids from 2-12 carbon atoms were better utilized by the animals than those of higher fatty acids such as palmitic, stearic, or oleic acids, and also that the mixture of free fatty acids and glycerin was inferior to the corresponding glyceride in nutritive value. Such a difference was more pronounced when the rats were fed on the artificial diet poor in vitamin-A: i. e. solid fats such as palmitin, stearin etc. were very difficultly assimilated or sometimes even retarded the animal growth unless an enough amount of vitamin-A was not given with them; while the lower members such as caprylin, caprin, laurin or myristin etc. could be more easily utilized and resulted a good animal growth even if the diet was deficient in vitamin-A. This evidence is especially interesting on account of that butter fat consists of mostly lower glycerides : those of from butyric to lauric acids ; and those of oleic and palmitic acids, etc. are present in it only in negligible quantity. Takahashi further observed that the rats could attain the normal 
growth with a fat-free diet, provided other nutritive factors were supplied in adequate proportion. Thus, the fat can be considered as not an indispensable food factor, at least, for a certain kind of animals.

The author has carried out a series of feeding experiments with the synthetical fats which were prepared from fatty acids containing uneven-number carbon atoms for the purpose of determining whether they could equally be well utilized by animals as in the case of those of even-number carbon atoms.

From the clinical stand-point of diabetes this subject is also of some interest, for it is now believed by some authors that synthetic margarin (intervin) prepared from margaric acid of 17 carbon atoms may be recommended for diabetes; and the synthetic margarin does not, according to these authors, produce the noxious "aceton bodies", which fact can be easily understood by the Knoop's " $\beta$-oxidation theory". On the other hand, others think another noxious product such as propionic or valerianic acid etc. might be produced from "intervin" instead of "aceton bodies". Therefore, careful feeding experiments with pure synthetical fats are absolutely necessary for the solution of the question.

The fatty acids used by the author for the synthetical preparation of fats were formic, propionic, isovaleric, heptoic, palargonic, undecyleic, undecylic, tridecylic, pentadecylic and margaric acids. All these, except propionic and isovaleric acids, were prepared by the author and they are carefully purified, and their melting- and boiling-points, mean molecular weights etc. were all examined thoroughly.

The preparation of triglycerides from these fatty acids was carried out as follows:- A calculated amount of pure glycerin was mixed with a little excess of fatty acid; to this mixture was added $5 \%$ of Twitschell's reagent, and heated at $100^{\circ} \mathrm{C}$ for about five hours, stirring the mixture occasionally in order to expell off the water produced by the reaction; the mixture gas dissolved in ether when the reaction was completed and carefully washed with dilute alkali, acid and water, successively. The etherial solution, after dehydrating with dry sodium sulphate, was evaporated, and the residue was either subjected to fractional distillation in vacuum or recrystallized from alcohol or ether, according to the nature of fats.

The basal diet for the albino rats consisted of $80 \%$ starch, $15 \%$ meat protein, 5\% McCollum's salt mixture, 3\% commercial "Oryzanin" and $0.1 \%$ "Biosterin". Young rats were previously fed on a limited quantity of the above diet ( 9 grams in dry matter for a rat per day), having each one been kept in 
a separate cage; and thus the animals grew at first to a certain degree, but they soon reached to the equilibrium state when the given amount of the diet was just sufficient for the maintenance of the body weight. At this point the synthetical fats tested were added to the above diet in the proportion of 5,10 , or $20 \%$. If the fats intaken were utilized by the rats, the animals would begin to grow, but in case they were noxious or quite useless for the animals the body weight would either decrease or at least remain constant. So, by plotting the body weight in curves the relative nutritive value of these fats can be easily shown. For control's sake, the parallel experiments with the fats of even-number carbon atoms such as acetin, butyrin, caprylin etc. were carried out under the same condition as above. The results obtained in this way are given below according to the order of their nutritive value.

1. By adding $5 \%$ fats to the basal diet :

palmitin, caprylin, acetin, heptylin, butyrin, undecylin, nonylin, pentadecylin, tridecylin, margarin, stearin, undecylein, propionin, valerin.

2. By adding $10 \%$ fats :

caprylin, heptylin, butyrin, pentadecylin, palmitin, tridecylin, stearin, acetin, nonylin, margarin.

3. By adding $20 \%$ fats :

tridecylin, pentadecylin, heptylin, stearin.

Among these, propionin, isovalerin and undecylein exhibited a noxious effect when $5 \%$ of these was added to the basal diet given. By decreasing this additional amount to $2.7 \%$, propionin and undecylein were found to be nutritious, while isovalerin was still noxious. It is found by the author that the last substance was the most noxious one among the above fats. Undecylin prepared by means of the catalytic reduction of undecylein was proved to be nutritious when an extent of $5 \%$ of this substance present in the diet. Therefore the noxious property of undecylein may be due to the presence of double linking in its molecule.

The experimental results given above are considered as most probably presenting only an approximate value, for the animal growth is much influenced not only by the room temperature, but also by the different seasons in which the feeding experiments are made.

The author also observed that the addition of vitamin $\mathrm{C}$ in these experiments gave not any noticeable effect upon the results. 
Showing the increase of body weight after adding $5 \%$

$$
\text { of sample to the basal diet. }
$$

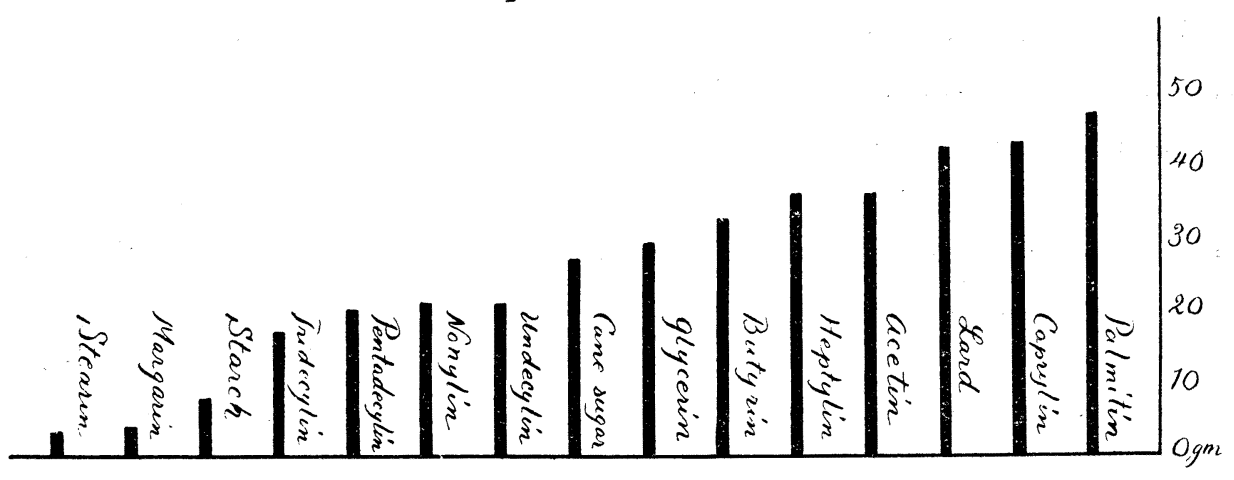

Showing the increase of body weight after adding 10\%

of sample to the basal diet.

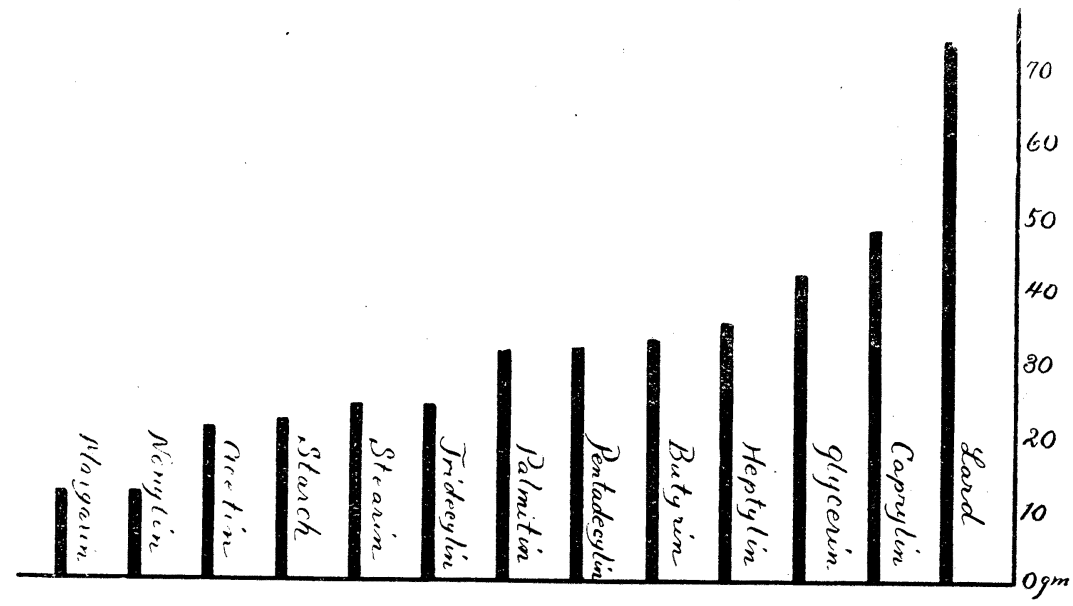

Oxiditative Nature of Iron Compounds. Part II.

Ferric ferricyanide as an oxidizing agent of organic compounds.1

By Bunsuke Suzuki and Tsutomu Maruyama.

(Recieved, Nov. 12, 1925)

Ferric ferricyanide is an good oxidizing reagent for $\alpha$-hydroxy- and $\alpha$-ketonic acids of aliphatic series, giving aldehydes and ketones as products, upon which as well as upon alcohols, ketones, sugars and $\alpha$-amino acids, the reagent has no 